\title{
Optimization of Radiation Dose and Image Quality for Large Field of View Cone-beam Computed Tomography: An In Vitro Study
}

\author{
Abeer A Almashraqi
}

\begin{abstract}
Aim and objective: This study aims to optimize the radiation dose and the image quality of a large field of view (FOV) cone-beam computed tomography (CBCT).

Materials and methods: Effective dose (E) and image quality delivered by six protocols that varied according to voxel size (VS) (0.25, 0.3 , and $0.4)$ and exposure time (ET) $(7,4$, and 2 seconds) were compared by scanning a large FOV $(16 \times 13 \mathrm{~cm})$ of a RANDO phantom using CBCT and each protocol was scanned twice. Thermoluminescent dosimeters placed on the phantom head were used to calculate E. Image noise and subjective image assessment were utilized to quantitatively and qualitatively assess the image quality, respectively. Repeated-measures analysis of variance (ANOVA) was used for comparisons with a significant level set at $\leq 0.05$.

Results: All protocols generated significantly different Evalues compared with each other ( $p$ values ranged between $<0.0001$ and $<0.05$ ) except for protocols V and VI $(p>0.05)$. Regarding image quality, both image noise and subjective assessment revealed that protocol V had acceptable image quality when compared with the standard protocol.

Conclusion: The use of 0.3 VS together with the lowest ET, as recommended by the manufacturer, can minimize the radiation dose while maintaining the diagnostic image quality generated from a large-FOV CBCT.

Clinical significance: Balancing between radiation dose reduction and diagnostic image quality is an important issue in diagnostic imaging that maximizes the clinical benefits and decreases the patient risks of ionizing radiation, especially for a large-FOV CBCT.

Keywords: Cone-beam computed tomography, Dose reduction, Effective dose, Image noise, Subjective image assessment.

World Journal of Dentistry (2020): 10.5005/jp-journals-10015-1768
\end{abstract}

\section{INTRODUCTION}

Since its introduction in 1998, cone-beam computed tomography (CBCT) has become one of the most important imaging modalities in dentistry. This advanced imaging technique delivers more spatial resolution and less radiation compared with its alternative multidetector computed tomography (MDCT). ${ }^{1,2}$ However, the radiation dose is still higher than the conventional radiographic techniques, which highlights the particularly pertinent need for dose optimization. Cone-beam computed tomography is used in many dentistry disciplines: Implantology, orthodontics, periodontology, pediatric dentistry, endodontics, and maxillofacial surgery. ${ }^{3-7}$

Radiation dose can be expressed in effective dose (E), equivalent dose, and absorbed dose. Effective dose is the most appropriate but it cannot be quantified directly in patients and must be measured either by in vitro studies or computer modeling. ${ }^{8}$ To calculate the radiation risk, the total effective dose received by all sensitive organs must be specifically and accurately measured. The radiation exposure of $\mathrm{CBCT}$ is affected by different factors including tube voltage, tube current, exposure time (ET), voxel size (VS), and field of view (FOV). These factors are changed according to the diagnostic tasks. ${ }^{9,10}$ Because of the widespread use of $C B C T$, many machines are available with different specifications. Moreover, many studies have focused on dose optimization using various non-standardized subjects including skulls, patient data, and anthropomorphic phantoms. ${ }^{11-13}$ The variation in CBCT imaging parameters, machines, and subjects makes optimizing diagnostic procedures difficult and standardizing these protocols even more challenging.
Department of Maxillofacial Surgery and Diagnostic Sciences, College of Dentistry, Jazan University, Jazan, Kingdom of Saudi Arabia; Department of Oral Radiology, Faculty of Dentistry, Ibb University, Ibb, Republic of Yemen

Corresponding Author: Abeer A Almashraqi, Department of Maxillofacial Surgery and Diagnostic Sciences, College of Dentistry, Jazan University, Jazan, Kingdom of Saudi Arabia; Department of Oral Radiology, Faculty of Dentistry, Ibb University, Ibb, Republic of Yemen, Phone: +966502210865, e-mail: abeerradiology@gmail.com

How to cite this article: Almashraqi AA. Optimization of Radiation Dose and Image Quality for Large Field of View Cone-beam Computed Tomography: An In Vitro Study. World J Dent 2020;11(5):346-354.

Source of support: Nil

Conflict of interest: None

Cone-beam computed tomography procedures are optimized by minimizing radiation dose while maintaining the diagnostic image quality, which can be accomplished by adjusting certain CBCT parameters. Image quality can be assessed using subjective or objective methods and the latter is usually performed on standardized test phantoms. These phantoms enable systematic and empirical quantification of the physical factors known to directly affect the image quality including image noise, contrast resolution, and spatial resolution. ${ }^{14-16}$ To measure these factors effectively and ethically, standardized phantoms are essential.

While many studies have been dedicated to reducing radiation dose during the large-FOV CBCT acquisitions, none of these also 
considered image quality. ${ }^{17-23}$ The recent systematic review of da Silva Moura et al. ${ }^{24}$ conducted on the factors influencing the effective dose associated with $C B C T$, recommended the need for more studies focusing on both the image quality requirements and the radiation dose measurement on CBCT machines. On the contrary, the studies that do correlate radiation dose with image quality focused on the optimization from the point of view of FOV and recommended the use of small FOV to optimize the radiation dose. ${ }^{10,25,26}$ However, there are diagnostic tasks that require using a large FOV, such as, for orthodontics, maxillofacial trauma, orthognathic surgery, extensive pathologies, and placing multiple implants in both arches; as such, the optimization of CBCT parameters for large FOVs is particularly pressing. The current study aimed to optimize the acquisition parameters of ET and VS toward minimizing radiation dose while maintaining the acceptable diagnostic image quality based on the standard protocol recommended by the manufacturer during a large-FOV CBCT diagnostic imaging.

\section{Materials and Methods}

\section{Cone-beam Computed Tomography Machine and Parameters}

i-CAT Next Generation (Imaging Sciences International, Hatfield, Pennsylvania, USA) CBCT machine was used to scan a $16 \times 13 \mathrm{~cm}$ FOV from the nasion to the end of the lower jaw. Six protocols (I-VI) were run that varied according to ET (pulsed time) and VS, as recommended by the manufacturer. The only exception was protocol II, which had lower ET than that suggested by the manufacturer (Table 1). Each protocol was scanned twice and they were ordered from I to VI according to the measured radiation dose and protocol I was the standard protocol for large FOV.

\section{Anthropomorphic Phantom and Thermoluminescent Dosimeters Chip Placement}

The adult male Alderson RANDO phantom (Alderson Research Laboratories Inc., Stamford, Connecticut, USA) was used in the current study. This anthropomorphic head and neck model consists of a human skull immersed in isocyanic rubber to mimic human soft tissues divided into 10 (2.5-cm thick) transverse slices and holes that represent different sensitive organs in each slice. Radiation doses were recorded using thermoluminescent dosimeters chips (TLDs) [LiF:Mg-Ti (TLD-700) with a diameter of $4.5 \mathrm{~mm}$ and a thickness of $0.9 \mathrm{~mm}$ ] that loaded in the holes of different sensitive organs found in each slice. Fourteen sites corresponding to 10 sensitive organs were selected on the phantom for TLD placement; brain, eye (inner and outer canthi), three sites on skin (midline of the neck, cheek, and nasion), four sites representing bone marrow (body and ramus of the mandible, cervical spine, and calvarium), thyroid gland, three salivary glands, and remainder tissues. Four tissues were measured to calculate radiation dose of remainder tissue; extrathoracic airway, oral mucosa, lymph nodes, and muscles in which their sites were measured in the previous sensitive organs. After inserting three TLD chips at each site ( 42 total TLDs in all sites), the head sections of the phantom were reassembled, screwed together tightly, and, at this point, ready to be scanned.

\section{Data Acquisition}

The TLD-loaded anthropomorphic phantom was positioned on the $\mathrm{CBCT}$ machine. The position of the phantom was standardized using a laser beam to center the phantom, so its midsagittal plane was perpendicular to the floor and its Frankfort plane was parallel to the floor. Each scanning protocol was performed twice at two different intervals to ensure the reliability of the measurements. Also, the TLD-loaded phantom head was exposed twice during each scan for every protocol to ensure signal detection even from low radiation doses and the resulting value was divided by 2 .

\section{Effective Dose Measurement}

The TLDs were calibrated to measure their sensitivity by exposing them to a known amount of radiation. An automatic hot gas reader (the Alnor Dosacus TLD-reader system, Finland) was used to analyze the TLDs. A sensitivity calculation was performed by dividing the reader's value for the amount of radiation of exposed TLD by the known amount of radiation to determine the calibration factor used to calibrate the TLD readings for the following exposures.

Then, the Alnor Dosacus TLD-reader system was used to read the dose values of the TLDs within 24 hours of exposure. The values were recorded for each TLD, the calibration factor was applied, and the values from the three chips from each site were averaged to obtain the dose absorbed at each site in micrograys ( $\mu \mathrm{Gy}$ ). The average absorbed dose of each site (i.e., organ) was further divided by 2 to compensate for the two exposures delivered for each scan. The standard deviation of the TLD-700 readings was $\leq 5 \%$.

The equivalent dose $\left(H_{\mathrm{T}}\right)$ was calculated using the following equation: ${ }^{27}$

$$
H_{\mathrm{T}}=\sum W_{\mathrm{R}} \times D_{\mathrm{T}} \text {, }
$$

in which $D_{\mathrm{T}}$ is the absorbed dose of each organ and $W_{\mathrm{R}}$ is the radiation-weighting factor ( $W_{R}$ is 1 in X-radiation). The determination of the $H_{T}$ is based on the distribution of these tissues in the head and neck region throughout the adult body (Online Table 1). In the present study, the calculations of $H_{\mathrm{T}}$ followed that of Ludlow et al. ${ }^{17,18}$ The $H_{\mathrm{T}}$ for bone marrow follows the distribution of active bone marrow throughout the adult body and was calculated using the summation of the individual $H_{\mathrm{T}}$ to the calvarium (11.8\%), mandible (1.3\%), and cervical spine (3.4\%). The $H_{\mathrm{T}}$ for the bone surface was calculated as: $H_{\mathrm{T}}$ of bone marrow $\times 4.64 .{ }^{17,18}$ However,

Table 1: Settings of the cone-beam computed tomography protocols

\begin{tabular}{llllll}
\hline Protocol no. & FOV & KV & $m A$ & $E T$ & 7 \\
I & $16 \times 13$ & 120 & 5 & 4 & 0.25 \\
II & $16 \times 13$ & 120 & 5 & 4 & 0.25 \\
III & $16 \times 13$ & 120 & 5 & 4 & 0.3 \\
IV & $16 \times 13$ & 120 & 5 & 2 & 0.4 \\
V & $16 \times 13$ & 120 & 5 & 2 & 0.3 \\
VI & $16 \times 13$ & 120 & 5 & 0.4 \\
\hline
\end{tabular}

FOV, field of view; $\mathrm{KV}$, kilovoltage, $\mathrm{mA}$, milliamperage; $\mathrm{ET}$, exposure time (pulsed time) in seconds; VS, voxel size in $\mathrm{mm}^{3}$ 
the proportion of the skin surface area, lymphatic nodes, and muscles in the head and neck region were estimated as $5 \%$ of the total body and accordingly the $H_{\mathrm{T}}$ of these tissues were calculated as the mean absorbed dose of each tissue $\times 5 \%$.

The effective dose was determined according to the ICRP 2007 and the following equation: ${ }^{28}$

$$
E=\sum W_{\mathrm{T}} \times H_{\mathrm{T}}
$$

in which $W_{\mathrm{T}}$ is the relative participation of that organ or tissue to the whole risk as shown in the Online Table 2. The overall risk to the body was determined by summing the effective doses of all exposed organs and tissues.

\section{Image Quality Evaluation}

Image quality was assessed by measuring image noise, represented by the standard deviation of the water's radiographic density. A water phantom (cylindrical water-filled Perspex) was scanned to measure the image noise in the six $\mathrm{CBCT}$ protocols. After scanning, the image noise was determined using the "regions of interest" tool in the i-CAT software without applying filter algorithms. This tool was used to select a rectangular area of $110 \mathrm{~cm}^{2}$ from the middle of the image in three different sections for each protocol. Next, the water's radiographic density was measured in mean, max, min, and standard deviation. The standard deviation signified the number of random fluctuations in CBCT number and represented image noise; the image noise is directly proportional to the standard deviation. ${ }^{28}$ The results of the image noises of the different protocols were compared with that of the standard protocol (protocol I).

After scanning of the RANDO phantom, the data set of each protocol were stored as DICOM format and projected on $3 \mathrm{D}$ workstation for evaluation. Subjective assessment of the image quality was conducted by three oral and maxillofacial radiologists with professional experience of more than 10 years. Prior to the image evaluation, inter- and intra-rater reliabilities of the three radiologists were calculated. The evaluation was carried out independently and blindly to the exposure parameters of each protocol. The three evaluators were asked to assess the overall diagnostic image quality (degree of sharpness and clarity of details) of the different protocols in comparison to the baseline images of the standard protocol (protocol I). The scoring was performed from 1 to 4 as follow; ${ }^{29} 1$ = very good, $2=$ good, $3=$ acceptable, $4=$ unacceptable (Figs 1 to 3 ).

\section{Statistical Analysis}

Raw data were imported into IBM ${ }^{\otimes}$ SPSS $^{\oplus}$ Statistics for Windows, version 21 (IBM Corp, Armonk, New York, USA) to be visualized, explored, and analyzed. $E$ and image noise are presented as means and standard deviations. These two variables were compared across the six CBCT protocols by repeated-measures analysis of variance (ANOVA) and Bonferroni's post hoc test was used for pair-wise comparisons. A $p$ value $\leq 0.05$ was considered statistically significant. Inter- and intra-rater reliabilities were calculated using Kappa statistic.

\section{Results}

The mean effective doses for the six CBCT protocols are presented in Table 2 according to organ. The highest dose was for the remainder tissues followed by the salivary glands and the thyroid gland. Skin received the lowest dose followed by the bone surface. For all organs, higher VS and lower ET decreased the effective dose with the highest E observed with the standard protocol I (0.25 VS, 7s ET,
$E=191.22 \mu \mathrm{SV})$ and the lowest with protocol VI (0.4 VS, 2s ET, $E=$ $68.4 \mu \mathrm{Sv}$ ). Protocols III, IV, V, and VI had a dose reduction of 36,40 , 60 , and $64.2 \%$ from protocol I, respectively.

Comparing the total effective doses of the six $\mathrm{CBCT}$ protocols revealed statistically significant differences between all protocols $(p<0.001$ or $p<0.05$ ) except between protocols V and VI. Regarding the specific organs, the remainder tissues received significantly different doses in all protocols $(p<0.001$ or $p<0.01)$ and the salivary glands showed statistically significant differences among all protocols (range between $p<0.05$ to $p<0.001$ ) except between protocols V and VI. All of the other organs showed statistically significant differences across all protocols (range between $p<0.05$ to $p<0.001$ ) except between protocols III and IV and protocols $\mathrm{V}$ and VI (Table 3).

The mean image noise values for the six CBCT protocols indicated that the most image noise appeared in protocol II followed by protocols VI (112.19 and 81.74, respectively) and the lowest image noise was seen in protocol IV followed by protocols III and V (54.88, 56.38, and 75.53, respectively) (Table 4).

As detailed in Table 5, comparing the image noise of the six CBCT protocols revealed statistically significant differences between protocol II and all other protocols $(p<0.001$ or $p<0.01)$. However,

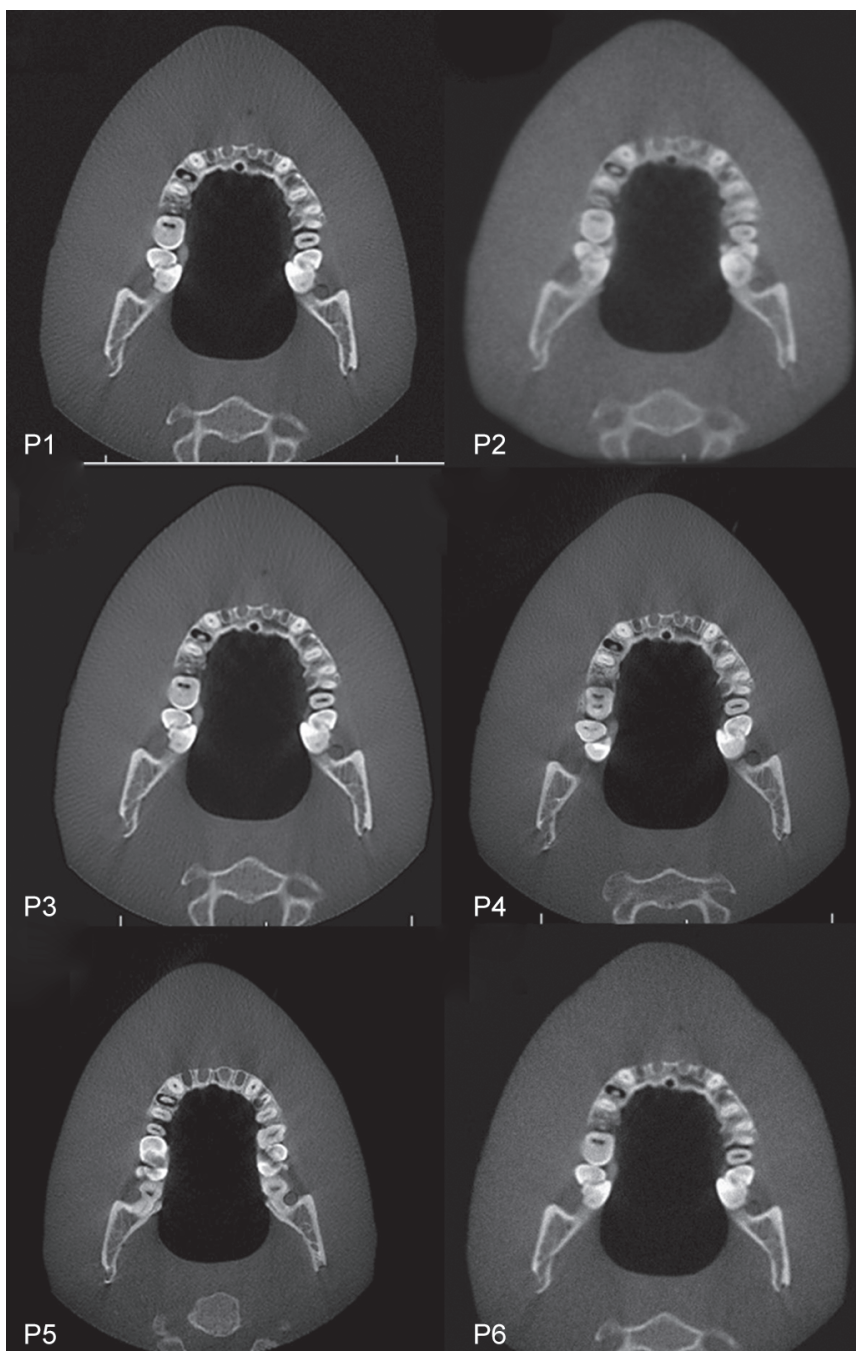

Fig. 1: Axial sections of different cone-beam computed tomography protocols: P1, protocol I; P2, protocol II; P3, protocol III; P4, protocol IV; P5, protocol V; P6, protocol VI 


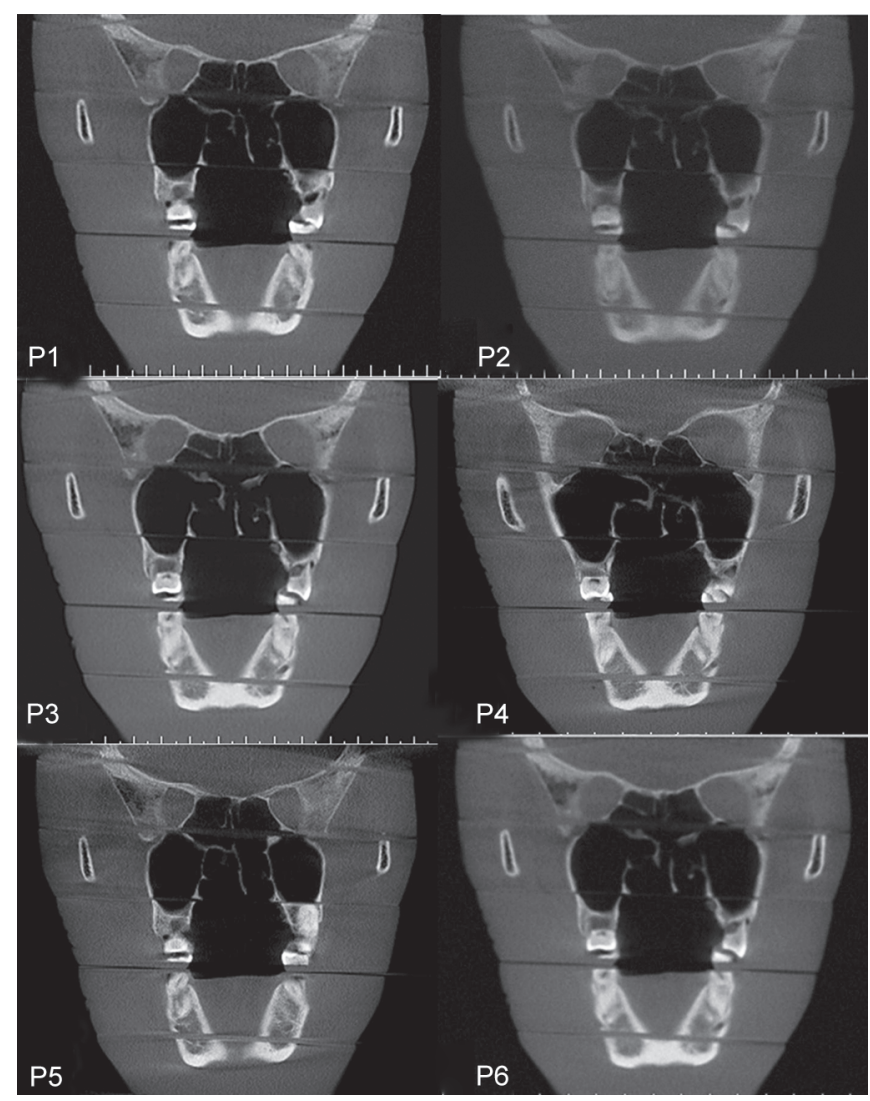

Fig. 2: Coronal sections of different cone-beam computed tomography protocols: P1, protocol I; P2, protocol II; P3, protocol III; P4, protocol IV; $\mathrm{P} 5$, protocol V; P6, protocol VI

the standard protocol I was not statistically different from protocols $\mathrm{V}$ and $\mathrm{VI}$, protocol IV was not statistically different from protocol III, and protocol V was not statistically different from protocols I, III, and VI. Additionally, Table 6 and Figures 1 to 3 revealed the results of the subjective image assessments conducted by three observers. The results showed good agreements between reviewers in which all of them agreed on the unacceptable image quality of protocol II and acceptable image quality for protocol VI. On the contrary, protocols III, IV, and V revealed a range of good to very good image quality when compared with the standard protocol (protocol I) with interand intra-rater reliabilities ranged from moderate (Kappa value of 0.568 ) to excellent (Kappa value of 1). Protocol V had an acceptable diagnostic image quality on both objective and subjective image quality assessments when compared with the standard protocol (protocol I).

\section{Discussion}

Optimization of radiation dose and image quality is an essential component of radiation protection that follows the ALADA principle; ${ }^{30}$ "as low as diagnostically acceptable". Cone-beam computed tomography is an important radiographic technique that is widely used in different diagnostic tasks in dentistry. While CBCT delivers a lower radiation dose than MDCT, this is still higher than conventional radiography, especially while imaging a large FOV. Using a small FOV is always preferable due to its lower radiation dose and higher image quality relative to a large $F O V .{ }^{10,25,26}$ However, some diagnostic tasks cannot be accomplished with a

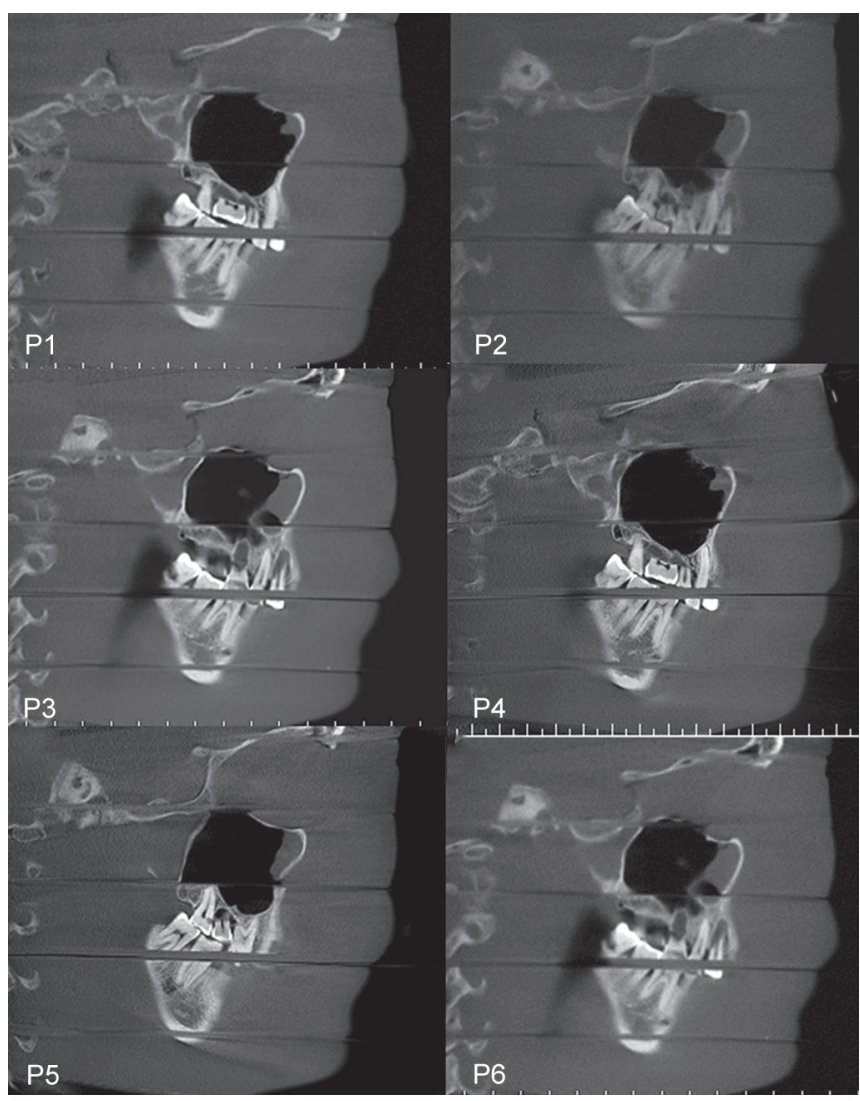

Fig. 3: Sagittal sections of different cone-beam computed tomography protocols: P1, protocol I; P2, protocol II; P3, protocol III; P4, protocol IV; $\mathrm{P} 5$, protocol V; P6, protocol VI

small FOV, e.g., orthognathic surgery, orthodontics, maxillofacial surgery, diagnosis of large pathologies, and 3D reconstruction of the whole maxillofacial region (to optimize the treatment planning). Unfortunately, acquiring a large FOV is always accompanied by a higher radiation dose and a lower spatial resolution. The radiation dose delivered during large FOVs is the main concern that requires addressing while not sacrificing the diagnostic image quality.

The results of the present study demonstrated that radiation dose was significantly reduced using a higher VS and a lower ET without compromising image quality. These findings are in agreement with other studies conducted using different FOVs that concluded both higher VS and lower ET are associated with reduced radiation dose. $29,31,32$ In contrast to the current study, Sonya et al. ${ }^{10}$ found no association between radiation dose and VS or spatial resolution. This discrepancy can be attributed to inherent differences between the two studies in terms of radiation dose measurements, which were measured on the center of the phantom not on sensitive organs and also they used almost identical imaging parameters in their study.

The total effective dose delivered during the CBCT protocols in the present study ranged from 191.22 to $68.4 \mu \mathrm{Sv}$ with the highest value observed in the standard protocol I ( $0.25 \mathrm{VS}, 7 \mathrm{~s} \mathrm{ET)}$ and the lowest in protocol VI ( $0.4 \mathrm{VS}, 2 \mathrm{~s}$ ET). These results are comparable to the findings of the previous studies used anthropomorphic phantoms and the same FOV on the same CBCT machine as the study of Ludlow et al. ${ }^{18}$ who recorded E values of 193.4 and 104.5 $\mu$ Sv (37.5 and $22.9 \mathrm{mAs}$, respectively), which are comparable 


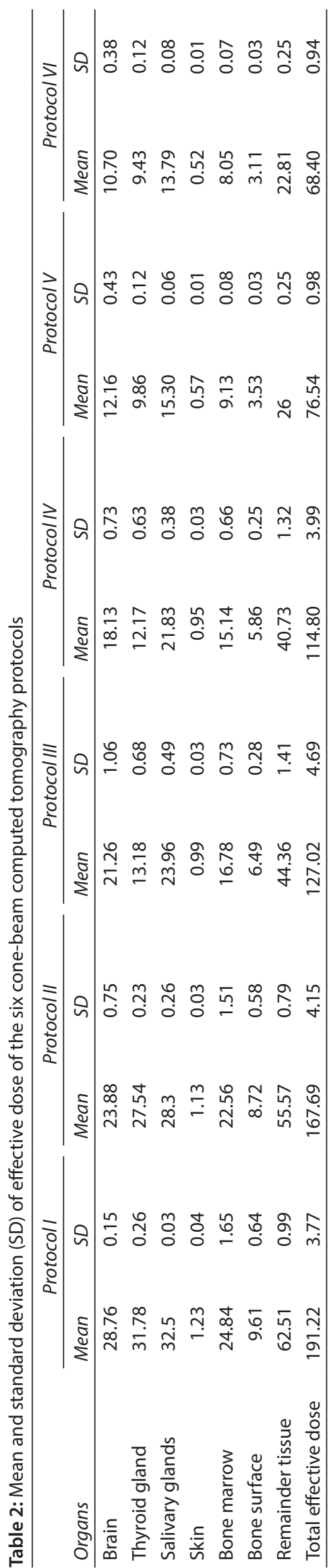

to the results of protocols I and IV, and the Roberts et al. ${ }^{21}$ study which detected a comparable result to that of protocol IV (E value of $110.5 \mu \mathrm{Sv}$ with $0.4 \mathrm{VS}$ ). Additionally, Davies et al. ${ }^{22}$ used anthropomorphic phantoms and the same FOV on the same CBCT machine to generate $\mathrm{E}$ of $77 \mu \mathrm{Sv}(2.1 \mathrm{~mA}, 0.4 \mathrm{VS}$, and $4 \mathrm{~s} \mathrm{ET})$ which is almost the same as the result of protocol V. On the contrary, the results of the present study are higher than the study of Ludlow and Ivanovic ${ }^{20}$ and Pauwels et al. ${ }^{23}$ who measured the E using the same $\mathrm{CBCT}$ machine and FOV of the current study and they found $E$ value of $87 \mu \mathrm{Sv}(19 \mathrm{mAs}$, VS $0.2-0.4)$ and $83 \mu \mathrm{Sv}$ (18.5 mAs), respectively. This variation is likely found on the results of the radiation dose of the bone surface and reminder tissues, which could be ascribed to the different methods used for the calculation.

Image noise was utilized in the current study as a quantitative measure of image quality and, as such, was used to compare the six CBCT protocols. The physical parameters that most impact the quality of clinical images are spatial resolution, contrast resolution, and image noise. ${ }^{14-16}$ In CBCT, contrast resolution is low and spatial resolution is most critical in diagnostic tasks that require high levels of detail, which are usually best captured using small FOVs. Therefore, image noise, which is affected by FOV, VS, and $\mathrm{mAs}$ [tube current (mA) and ET (s)], ${ }^{32-34}$ is a suitable physical factor to quantitatively assess the quality of large-FOV images. However, some studies ${ }^{16,34,35}$ recommend measuring the modulation transfer function and/or contrast-to-noise ratio because these significantly associate with subjective image quality; i.e., these physical factors strongly correlated with the ability to effectively accomplish specific diagnostic tasks and, therefore, should serve as reliable, objective indicators of image quality. Additionally, image noise has an effect on the contrast-to-noise ratio of an image and the highest the image noise is the lowest the contrast-to-noise ratio. ${ }^{16,32}$ Because the image noise alone was not enough to assess the image quality, the subjective assessment was performed in the current study taken into account the images of the standard protocol as the baseline for assessment.

The results of the present study indicated that image noise decreased as VS and ET increased. There was no statistically significant difference between the standard protocol I and protocol V or VI and between protocol III and protocol IV or V but protocol II was significantly different from all of the other protocols. This finding is consistent with Almashraqi et al. ${ }^{29}$ who found that image noise is inversely proportional to VS and $\mathrm{mAs}$. In accordance with the image noise, the subjective assessment of image quality with good agreements between observers also revealed that protocols III, IV, and V had a range of a good to very good image quality in relation to the standard protocol (protocol I), while protocol II had unacceptable image quality. This is because smaller VSs need longer ETs to decrease image noise and, accordingly, to improve image quality. This supports other studies that concluded lower VSs require more exposure to achieve high image quality by decreasing image noise and increasing spatial resolution. ${ }^{31,36,37}$ It should be also noted that the main purpose of using protocol II in the present study, used 0.25 VS with lower ET than that recommended by the manufacturer, was to investigate how much variation can occur on the level of radiation dose and image quality and whether this variation is beneficial or not. Based on the results of the current study, protocol II demonstrated the highest image noise and the lowest diagnostic image quality with only $12 \%$ radiation dose reduction from the standard protocol which indicated the importance of radiation dose optimization within the recommendations of the manufacturer. 
Table 3: Mean difference and significant level of radiation doses in different tissues in the six cone-beam computed tomography protocols using repeated measures ANOVA

\begin{tabular}{|c|c|c|c|c|c|c|}
\hline Protocol & Protocoll & Protocol II & Protocol III & Protocol IV & Protocol V & Protocol VI \\
\hline \multicolumn{7}{|l|}{ Brain } \\
\hline Protocol I & NA & $4.88^{* *}$ & $7.50^{* *}$ & $10.63^{* * *}$ & $16.60^{* * *}$ & $18.06^{* * *}$ \\
\hline Protocol II & - & NA & 2.62 & $5.75^{* *}$ & $11.72^{* * *}$ & $13.18^{* * *}$ \\
\hline Protocol III & $7.50^{* *}$ & 2.62 & NA & 3.13 & $9.10^{* * * *}$ & $10.56^{* * *}$ \\
\hline Protocol IV & $10.63^{* * *}$ & $5.75^{* *}$ & 3.13 & NA & $5.97^{* *}$ & $7.43^{* *}$ \\
\hline Protocol V & $16.60^{* * *}$ & $11.72^{* * *}$ & $9.10^{* * *}$ & $5.97^{* *}$ & NA & 1.45 \\
\hline Protocol VI & $18.06^{* * *}$ & $13.18^{* * *}$ & $10.56^{* * *}$ & $7.43^{* *}$ & 1.45 & NA \\
\hline \multicolumn{7}{|l|}{ Thyroid } \\
\hline Protocol I & NA & $4.24^{* * * *}$ & $18.60^{* * *}$ & $19.61^{* * *}$ & $21.92^{* * *}$ & $22.35^{* * *}$ \\
\hline Protocol II & $4.24^{* * * *}$ & NA & $14.36^{* * *}$ & $15.37^{* * * *}$ & $17.68^{* * *}$ & $18.11^{* * *}$ \\
\hline Protocol III & $18.60^{* * *}$ & $14.36^{* * *}$ & NA & 1.01 & $3.32^{* * * *}$ & $3.75^{* * *}$ \\
\hline Protocol IV & $19.61^{* * *}$ & $15.37^{* * *}$ & 1.01 & NA & $2.31^{* *}$ & $2.74^{* *}$ \\
\hline Protocol V & $21.92^{* * *}$ & $17.68^{* * *}$ & $3.32^{* * * *}$ & $2.31^{* *}$ & NA & 0.42 \\
\hline Protocol VI & $22.35^{* * *}$ & $18.11^{* * *}$ & $3.75^{* * *}$ & $2.74^{* *}$ & 0.42 & NA \\
\hline \multicolumn{7}{|l|}{ Salivary glands } \\
\hline Protocol I & NA & $4.20^{* *}$ & $8.53^{* * * *}$ & $10.67^{* * *}$ & $17.19^{* * *}$ & $18.71^{* * *}$ \\
\hline Protocol II & $4.20^{* *}$ & NA & $4.33^{* *}$ & $6.47^{* * *}$ & $12.99^{* * *}$ & $14.51^{* * *}$ \\
\hline Protocol III & $8.53^{* * *}$ & $4.33^{* *}$ & NA & $2.13^{*}$ & $8.66^{* * *}$ & $10.17^{* * *}$ \\
\hline Protocol IV & $10.67^{* * *}$ & $6.47^{* * *}$ & $2.13^{*}$ & NA & $6.52^{* * *}$ & $8.04^{* * * *}$ \\
\hline Protocol V & $17.19^{* * *}$ & $12.99^{* * *}$ & $8.66^{* * *}$ & $6.52^{* * *}$ & NA & 1.51 \\
\hline Protocol VI & $18.71^{* * *}$ & $14.51^{* * *}$ & $10.17^{* * *}$ & $8.04^{* * *}$ & 1.51 & NA \\
\hline \multicolumn{7}{|l|}{ Skin } \\
\hline Protocol I & NA & 0.10 & $0.24^{* *}$ & $0.28^{* *}$ & $0.66^{* * *}$ & $0.71^{* * *}$ \\
\hline Protocol II & 0.10 & NA & 0.14 & $0.18^{*}$ & $0.56^{* * *}$ & $0.61 * * *$ \\
\hline Protocol III & $0.24^{* *}$ & 0.14 & NA & 0.04 & $0.42^{* * * *}$ & $0.47^{* * *}$ \\
\hline Protocol IV & $0.28^{* *}$ & $0.18^{*}$ & 0.04 & NA & $0.38^{* * *}$ & $0.43^{* * *}$ \\
\hline Protocol V & $0.66^{* * *}$ & $0.56^{* * *}$ & $0.42^{* * *}$ & $0.38^{* * *}$ & NA & 0.05 \\
\hline Protocol VI & $0.71^{* * *}$ & $0.61^{* * * *}$ & $0.47^{* * *}$ & $0.43^{* * *}$ & 0.05 & NA \\
\hline \multicolumn{7}{|l|}{ Bone marrow } \\
\hline Protocol I & NA & 2.28 & $8.06^{* *}$ & $9.70^{* *}$ & $15.71^{* * * *}$ & $16.78^{* * * *}$ \\
\hline Protocol II & 2.28 & NA & $5.78^{*}$ & $7.42^{* *}$ & $13.43^{* * *}$ & $14.50^{* * *}$ \\
\hline Protocol III & $8.06^{* *}$ & $5.78^{*}$ & NA & 1.64 & $7.65^{* *}$ & $8.72^{* *}$ \\
\hline Protocol IV & $9.70^{* *}$ & $7.42^{* *}$ & 1.64 & NA & $6.01 *$ & $7.08^{* *}$ \\
\hline Protocol V & $15.71^{* * *}$ & $13.43^{* * *}$ & $7.65^{* *}$ & $6.01 *$ & NA & 1.07 \\
\hline Protocol VI & $16.78^{* * *}$ & $14.50^{* * * *}$ & $8.72^{* *}$ & $7.08^{* *}$ & 1.07 & NA \\
\hline \multicolumn{7}{|l|}{ Bone surface } \\
\hline Protocol I & NA & 0.88 & $3.11^{* *}$ & $3.75^{* *}$ & $6.07 * * *$ & $6.49 * * *$ \\
\hline Protocol II & 0.88 & NA & $2.23^{*}$ & $2.87^{* *}$ & $5.19^{* * * *}$ & $5.61^{* * *}$ \\
\hline Protocol III & $3.11^{* *}$ & $2.23^{*}$ & NA & 0.63 & $2.96^{* *}$ & $3.38^{* *}$ \\
\hline Protocol IV & $3.75^{* *}$ & $2.87^{* *}$ & 0.63 & NA & $2.32^{*}$ & $2.74^{* *}$ \\
\hline Protocol V & $6.07^{* * *}$ & $5.19^{* * *}$ & $2.96^{* *}$ & $2.32^{*}$ & NA & 0.42 \\
\hline Protocol VI & $6.49^{* * *}$ & $5.61^{* * *}$ & $3.38^{* *}$ & $2.74^{* *}$ & 0.42 & NA \\
\hline \multicolumn{7}{|c|}{ Remainder tissue } \\
\hline Protocol I & NA & $6.93^{* * *}$ & $18.14^{* * *}$ & $21.77^{* * *}$ & $36.50^{* * *}$ & $36.69^{* * *}$ \\
\hline Protocol II & $6.93^{* * *}$ & NA & $11.21^{* * *}$ & $14.84^{* * *}$ & $29.57^{* * *}$ & $32.76^{* * *}$ \\
\hline Protocol III & $18.14^{* * *}$ & $11.21^{* * *}$ & NA & $3.63^{* *}$ & $18.36^{* * *}$ & $21.55^{* * *}$ \\
\hline Protocol IV & $21.77^{* * *}$ & $14.84^{* * *}$ & $3.63^{* *}$ & NA & $14.73^{* * *}$ & $17.92^{* * * *}$ \\
\hline Protocol V & $36.50 * * *$ & $29.57^{* * *}$ & $18.36^{* * *}$ & $14.73^{* * *}$ & NA & $3.19^{* *}$ \\
\hline Protocol VI & $36.69^{* * *}$ & $32.76^{* * *}$ & $21.55^{* * *}$ & $17.92^{* * *}$ & $3.19^{* *}$ & NA \\
\hline
\end{tabular}


Contd...

\begin{tabular}{llllllc}
\hline Protocol & Protocol I & Protocol II & Protocol III & Protocol IV & Protocol V \\
\hline $\begin{array}{c}\text { Total effective dose } \\
\text { Protocol I }\end{array}$ & NA & $23.52^{* * *}$ & $64.19^{* * *}$ & $76.42^{* * *}$ & $114.68^{* * *}$ & $122.82^{* * *}$ \\
Protocol II & $23.52^{* * *}$ & NA & $40.67^{* * *}$ & $52.89^{* * *}$ & $91.15^{* * *}$ & $99.29^{* * *}$ \\
Protocol III & $64.19^{* * *}$ & $40.67^{* * *}$ & NA & $12.22^{*}$ & $50.48^{* * *}$ & $58.62^{* * *}$ \\
Protocol IV & $76.42^{* * *}$ & $52.89^{* * *}$ & $12.22^{* *}$ & NA & $38.25^{* * *}$ & $46.39^{* * *}$ \\
Protocol V & $114.68^{* * *}$ & $91.15^{* * *}$ & $50.48^{* * *}$ & $38.25^{* * *}$ & NA & 8.14 \\
Protocol VI & $122.82^{* * *}$ & $99.29^{* * *}$ & $58.62^{* * *}$ & $46.39^{* * *}$ & 8.14 & NA \\
\hline
\end{tabular}

${ }^{* * *} p<0.001,{ }^{* *} p<0.01,{ }^{*} p<0.05$

Table 4: Mean and standard deviation (SD) of objective assessment of the image quality in the six cone-beam computed tomography protocols

\begin{tabular}{lrl}
\hline Protocol & Mean & SD \\
\hline Protocol I & 76.84 & 2.93 \\
Protocol II & 112.19 & 8.47 \\
Protocol III & 56.38 & 7.01 \\
Protocol IV & 54.88 & 4.36 \\
Protocol V & 75.53 & 9.25 \\
Protocol VI & 81.74 & 5.21 \\
\hline
\end{tabular}

Table 5: Mean difference and significant level of objective assessment of the image quality in the six cone-beam computed tomography protocols using repeated measures ANOVA

\begin{tabular}{lllllll}
\hline Protocol & Protocoll & Protocol II & Protocol III & Protocol IV & Protocol V Protocol VI \\
\hline Protocol I & NA & $-35.35^{* * *}$ & $20.46^{*}$ & $21.96^{*}$ & 1.31 & -4.09 \\
Protocol II & $-35.35^{* * *}$ & NA & $55.81^{* * *}$ & $57.31^{* * *}$ & $36.66^{* * *}$ & $30.45^{* *}$ \\
Protocol III & $20.46^{*}$ & $55.81^{* * *}$ & NA & 1.51 & -19.15 & $-25.36^{* *}$ \\
Protocol IV & $21.96^{*}$ & $57.31^{* * *}$ & 1.51 & NA & $-20.65^{*}$ & $-20.87^{* *}$ \\
Protocol V & 1.31 & $36.66^{* * *}$ & -19.15 & $-20.65^{*}$ & NA & -6.21 \\
Protocol VI & -4.09 & $30.45^{* *}$ & $-25.36^{* *}$ & $-26.87^{* *}$ & -6.21 & NA \\
\hline
\end{tabular}

${ }^{* * *} p<0.001,{ }^{* *} p<0.01,{ }^{*} p<0.05$

Table 6: Subjective assessments of image quality of different cone-beam computed tomography protocols

\begin{tabular}{|c|c|c|c|c|c|c|}
\hline Observers & Protocoll & Protocol II & Protocol III & Protocol IV & Protocol V & Protocol VI \\
\hline First observer & 1 & 4 & 2 & 1 & 2 & 3 \\
\hline Second observer & 1 & 4 & 1 & 2 & 3 & 3 \\
\hline Third observer & 1 & 4 & 1 & 2 & 2 & 3 \\
\hline
\end{tabular}

Protocols VI, V, and IV decreased the effective dose by 64.2, 60 , and $40 \%$, respectively, relative to the standard protocol I. Moreover, protocol $\mathrm{V}$ generated acceptable diagnostic quality images in relation to the standard protocol on the level of both image noise and subjective image assessments. Therefore, protocol $V$ is best suited for a large-FOV CBCT imaging due to its optimized balance between ALADA principle of low radiation doses and good diagnostic-quality images.

The present study has its limitations. Due to the variation between $\mathrm{CBCT}$ machines, it would have been ideal to perform this optimization study on more than one CBCT machine; future studies should include multiple machines to ensure the broad applicability of the results. Another limitation is the use of only one objective metric for image quality assessment as it is preferable to conduct more than one metric. Future studies ought to include more CBCT machines in addition to another objective metric (e.g., modulation transfer function, contrast-to-noise ratio).

\section{Conclusion}

The use of 0.3 with the lowest ET, as recommended by the manufacturer, can minimize the radiation dose by $60 \%$ from the standard protocol, while maintaining the diagnostic image quality generated from a large-FOV CBCT scan. Further studies to optimize radiation dose and image quality of a large FOV using different CBCT machines with both subjective and objective image quality assessments are recommended.

\section{ACKnOWLedgments}

I graciously thank Dr Ali Gaber, Consultant Radiologist for his kind assistance in measuring image noise. I am very thankful to DrTenny John, Dr Sameena Parveen, and Dr Ankur Jethlia, Assistant Professors in Oral Medicine and Oral Radiology for their help in subjective assessments of the image quality of CBCT images. 


\section{References}

1. Hatcher DC. Operational principles for cone-beam computed tomography. J Am Dent Assoc 2010;141:3S-6S. DOI: 10.14219/jada. archive.2010.0359.

2. Morant J, Salvadó M, Hernández-Girón I, et al. Dosimetry of a cone beam CT device for oral and maxillofacial radiology using monte Carlo techniques and ICRP adult reference computational phantoms. Dentomaxillofac Radiol 2013;42(3):92555893. DOI: 10.1259/ dmfr/92555893.

3. Davies A, Patel S, Foschi F, et al. The detection of periapical pathoses using digital periapical radiography and cone beam computed tomography in endodontically retreated teeth-part 2: a 1 year posttreatment follow-up. Int Endod J 2016;49(7):623-635. DOI: 10.1111/ iej.12500.

4. Kapila S, Nervina J. CBCT in orthodontics: assessment of treatment outcomes and indications for its use. Dentomaxillofac Radiol 2015;44(1):20140282. DOI: 10.1259/dmfr.20140282.

5. Chan H-L, Misch K, Wang H-L. Dental imaging in implant treatment planning. Implant Dent 2010;19(4):288-298. DOI: 10.1097/ ID.0b013e3181e59ebd.

6. Bahadure R, Thosar N, Khubchandani M. Orthodontic extrusion: diagnosis and treatment with $C B C T$ in a pediatric patient. Gen Dent 2013;61(4):e5-e7.

7. Pour DG, Romoozi E, Shayesteh YS. Accuracy of cone beam computed tomography for detection of bone loss. J Dent 2015;12(7):513.

8. Fisher DR, Fahey FH. Appropriate use of effective dose in radiation protection and risk assessment. Health Phys 2017;113(2):102. DOI: 10.1097/HP.0000000000000674.

9. Pauwels R, Araki K, Siewerdsen J, et al. Technical aspects of dental CBCT: state of the art. Dentomaxillofac Radiol 2015;44(1):20140224. DOI: $10.1259 / \mathrm{dmfr} .20140224$.

10. Sonya DA, Davies J, Ford NL. A comparison of cone-beam computed tomography image quality obtained in phantoms with different fields of view, voxel size, and angular rotation for iCAT NG. J Oral Maxillofac Radiol 2016;4(2):31. DOI: 10.4103/2321-3841.183821.

11. Loubele $M$, Maes $F$, Jacobs $R$, et al. Comparative study of image quality for MSCT and CBCT scanners for dentomaxillofacial radiology applications. Radiat Prot Dosimet 2008;129(1-3):222-226. DOI: 10.1093/rpd/ncn154.

12. Suomalainen A, Kiljunen T, Kaser Y, et al. Dosimetry and image quality of four dental cone beam computed tomography scanners compared with multislice computed tomography scanners. Dentomaxillofac Radiol 2009;38(6):367-378. DOI: 10.1259/dmfr/15779208.

13. Liang $X$, Jacobs $R$, Hassan $B$, et al. A comparative evaluation of cone beam computed tomography (CBCT) and multi-slice CT (MSCT): part I. On subjective image quality. Eur J Radiol 2010;75(2):265-269. DOI: 10.1016/j.ejrad.2009.03.042

14. Vano E. ICRP recommendations on 'managing patient dose in digital radiology'. Radiat Prot Dosimetry 2005;114(1-3):126-130. DOI: 10.1093/ $\mathrm{rpd} / \mathrm{nch} 533$.

15. McCollough $\mathrm{CH}$, Bruesewitz MR, Mcnitt-Gray MF, et al. The phantom portion of the American College of Radiology (ACR) computed tomography (CT) accreditation program: practical tips, artifact examples, and pitfalls to avoid. Med Phys 2004;31(9):2423-2442. DOI: 10.1118/1.1769632.

16. ParkH-N, Min C-K, Kim K-A, et al. Optimization of exposure parameters and relationship between subjective and technical image quality in cone-beam computed tomography. Imaging Sci Dent 2019;49(2):139151. DOI: 10.5624/isd.2019.49.2.139.

17. Ludlow J, Davies-Ludlow L, Brooks S. Dosimetry of two extraoral direct digital imaging devices: NewTom cone beam CT and orthophos plus DS panoramic unit. Dentomaxillofac Radiol 2003;32(4):229-234. DOI: $10.1259 / \mathrm{dmfr} / 26310390$

18. Ludlow JB, Davies-Ludlow L, Brooks S, et al. Dosimetry of 3 CBCT devices for oral and maxillofacial radiology: CB mercuray, NewTom 3G and i-CAT. Dentomaxillofac Radiol 2006;35(4):219-226. DOI: 10.1259/ dmfr/14340323.
19. Silva MAG, Wolf $U$, Heinicke $F$, et al. Cone-beam computed tomography for routine orthodontic treatment planning: a radiation dose evaluation. Am J Orthod Dentofacial Orthop 2008;133(5):640. e1-5. DOI: 10.1016/j.ajodo.2007.11.019.

20. Ludlow JB, Ivanovic M. Comparative dosimetry of dental CBCT devices and 64-slice CT for oral and maxillofacial radiology. Oral Surg, Oral Med, Oral Pathol, Oral Radiol, Endodontol 2008;106(1):106-114. DOI: 10.1016/j.tripleo.2008.03.018.

21. Roberts J, Drage N, Davies J, et al. Effective dose from cone beam CT examinations in dentistry. Br J Radiol 2009;82(973):35-40. DOI: 10.1259/bjr/31419627.

22. Davies J, Johnson B, Drage N. Effective doses from cone beam CT investigation of the jaws. Dentomaxillofac Radiol 2012;41(1):30-36. DOI: $10.1259 / \mathrm{dmfr} / 30177908$.

23. Pauwels R, Beinsberger J, Collaert B, et al. Effective dose range for dental cone beam computed tomography scanners. Eur J Radiol 2012;81(2):267-271. DOI: 10.1016/j.ejrad.2010.11.028.

24. da Silva Moura W, Chiqueto K, Pithon GM, et al. Factors influencing the effective dose associated with CBCT: a systematic review. Clin Oral Investig 2019;23(3):1319-1330. DOI: 10.1007/s00784-0182561-4.

25. Hoffmann E, da Silva AM, Azevedo D, Towards Image Quality Analysis of Small and Full Field of View Dental Cone Beam CT Systems. Paper presented at: World Congress on Medical Physics and Biomedical Engineering, June 7-12, 2015, Toronto, Canada 2015.

26. Dillenseger J-P, Gros C-I, Sayeh A, et al. Image quality evaluation of small FOV and large FOV CBCT devices for oral and maxillofacial radiology. Dentomaxillofac Radiol 2017;46(1):20160285. DOI: 10.1259/ dmfr.20160285.

27. Protection ICoR. Recommendations of the International Commission on Radiological Protection. ICRP Publication 103. Ann ICRP 2007; 37(2-4):1-332.

28. Goldman LW. Principles of CT: radiation dose and image quality. J Nucl Med Technol 2007;35(4):213-225. DOI: 10.2967/jnmt.106.037846.

29. Almashraqi AA, Ahmed EA, Mohamed NS, et al. Evaluation of different low-dose multidetector CT and cone beam CT protocols in maxillary sinus imaging: part I - an in vitro study. Dentomaxillofac Radiol 2017;46(6):20160323. DOI: 10.1259/dmfr.20160323.

30. Jaju PP, Jaju SP. Cone-beam computed tomography: time to move from ALARA to ALADA. Imaging Sci Dent 2015;45(4):263-265. DOI: 10.5624/isd.2015.45.4.263.

31. Jadu FM, Alzahrani AA, Almutairi MA, et al. The effect of varying cone beam computed tomography image resolution and field-of-view centralization on effective radiation dose. Saudi Med J 2018;39(5):470. DOI: 10.15537/smj.2018.5.21658.

32. McGuigan M, Duncan H, Horner K. An analysis of effective dose optimization and its impact on image quality and diagnostic efficacy relating to dental cone beam computed tomography (CBCT). Swiss Dent J 2018;128(4):297-316.

33. Pauwels R, Seynaeve L, Henriques JCG, et al. Optimization of dental CBCT exposures through mAs reduction. Dentomaxillofac Radiol 2015;44(9):20150108. DOI: 10.1259/dmfr.20150108.

34. Al-Okshi A, Theodorakou C, Lindh C. Dose optimization for assessment of periodontal structures in cone beam CT examinations. Dentomaxillofac Radiol 2017;46(3):20160311. DOI: 10.1259/ dmfr.20160311.

35. Hidalgo Rivas JA, Horner K, Thiruvenkatachari B, et al. Development of a low-dose protocol for cone beam CT examinations of the anterior maxilla in children. Br J Radiol 2015;88(1054):20150559. DOI: 10.1259/ bjr.20150559.

36. Dong $\mathrm{T}$, Xia $\mathrm{L}, \mathrm{Cai} \mathrm{C}$, et al. Accuracy of in vitro mandibular volumetric measurements from CBCT of different voxel sizes with different segmentation threshold settings. BMC Oral Health 2019;19(1):206. DOI: 10.1186/s12903-019-0891-5.

37. Oenning AC, Pauwels $R$, Stratis $A$, et al. Halve the dose while maintaining image quality in paediatric cone beam CT. Sci Rep 2019;9(1):1-9. DOI: 10.1038/s41598-019-41949-w. 


\section{APPENDIX}

Appendix Table 1: Estimated percentage of tissue irradiated to calculate equivalent dose to a tissue or organ ${ }^{17,18}$

\begin{tabular}{lc}
\hline Sensitive organ & Irradiated fraction (\%) \\
\hline Brain & 100 \\
Eye & 100 \\
Skin & 5 \\
Bone marrow and bone surface $^{\dagger}$ & 16.5 \\
Mandible & 1.3 \\
Calvarium & 11.8 \\
Cervical spine & 3.4 \\
Salivary glands & 100 \\
Thyroid gland & 100 \\
Remainder tissues* & \\
a. Lymph nodes and muscles & 5 \\
b. Oral mucosa & 100 \\
c. Extrathoracic airway & 100 \\
\hline
\end{tabular}

*According to ICRP 2007

${ }^{\dagger}$ Equivalent dose of bone marrow $\times 4.64$
Appendix Table 2: Weighting tissue $\left(W_{T}\right)$ factors for calculation of effective dose ICRP $2007^{27}$

\begin{tabular}{ll}
\hline Sensitive organ & $W_{T} 2007$ \\
\hline Bone marrow & 0.12 \\
Breast & 0.12 \\
Colon & 0.12 \\
Lung & 0.12 \\
Stomach & 0.12 \\
Bladder & 0.04 \\
Esophagus & 0.04 \\
Gonads & 0.08 \\
Liver & 0.04 \\
Thyroid & $\mathbf{0 . 0 4}$ \\
Bone surface & $\mathbf{0 . 0 1}$ \\
Brain & $\mathbf{0 . 0 1}$ \\
Kidneys & - \\
Salivary glands & $\mathbf{0 . 0 1}$ \\
Skin & $\mathbf{0 . 0 1}$ \\
Remainder* & $\mathbf{0 . 1 2 *}$ \\
\hline
\end{tabular}

*Adrenals, extrathoracic region, gallbladder, heart, kidneys, lymphatic nodes, muscle, oral mucosa, pancreas, prostate, small intestine, spleen, thymus, and uterus/cervix. Italicized text represents remainder tissues used for calculation of maxillofacial dose

Bold terms represent the tissue or organ used for calculation in this study 